\section{Investigación sobre la transición entre las doctrinas mercantilistas y el nacimiento de la Economía Política. Un análisis de los aportes de Thomas Mun, James Steuart y David Hume ${ }^{1}$}

\section{Research on the transition between mercantilist doctrines and the birth of Political Economy. An analysis of the contributions of Thomas Mun, James Steuart and David Hume}

Recibido: 23 de marzo de 2016 - Revisado: 15 de abril de 2016 - Aceptado: 24 de junio de 2016.

Pilar Piqué2

Leandro Navarro ${ }^{3}$

Martín Harracá ${ }^{4}$

Pablo Benchimol ${ }^{5}$

Catalina Aldama ${ }^{6}$

\section{Resumen}

En el presente trabajo nos proponemos estudiar la progresión del pensamiento económico de los siglos XVII y XVIII, a partir del análisis de obras originales emblemáticas del período como las de Thomas Mun, James Steuart y David Hume. Indagaremos cómo estos autores comienzan a revelar el carácter fragmentario y limitado de las concepciones elaboradas por las doctrinas mercantilistas sobre los procesos económicos ante la emergencia y el desarrollo del capitalismo industrial. Asimismo, examinaremos cómo, en ese marco, surgen los esfuerzos de estas doctrinas económicas del siglo XVIII por entender los procesos económicos en un contexto universal y no unilateralmente nacional, pugnando por afianzar la conformación de un cuerpo teórico unificado del sistema capitalista como un todo, dando paso a la Economía Política.

\section{Palabras clave}

Historia del pensamiento económico, Ciencia Económica, Economía Política, teoría general de la reproducción capitalista, teoría general de la circulación mercantil, mercantilismo.

\begin{abstract}
In this article we study the progression of economic thought of the 17th and 18th centuries, based on the analysis of original emblematic works of the period such as Thomas Mun, James Steuart and David Hume. It is investigated how these authors begin to reveal the fragmentary and limited character of the conceptions elaborated by the mercantilist doctrines on the economic processes before the emergency and the development of industrial capitalism. It also examines how, in this context, the efforts of these economic doctrines of the eighteenth century arise from the understanding of economic processes in a universal and not unilaterally national context, striving to strengthen the formation of a unified theoretical body of the capitalist system as a whole, giving way to Political Economy.
\end{abstract}

\section{Keywords}

History of economic thought, economic science, political economy, general theory of capitalist reproduction, general theory of mercantilist circulation, mercantilism.
${ }^{1}$ Artículo elaborado en el marco del Proyecto de Investigación de Cátedra (PROINC) "Economía Política de la Poiesis. El potencial filosófico de la Teoría Económica", del Centro de Estudios para la Planificación del Desarrollo (CEPLAD), Instituto de Investigaciones Económicas (IIE), Facultad de Ciencias Económicas (FCE), Universidad de Buenos Aires (UBA). Financiado por la Universidad de Buenos Aires, Argentina.

${ }^{2}$ Lic. En Economía (UBA). Doctoranda en Economía (UBA). Becaria doctoral de Consejo Nacional de Investigaciones Científicas y Técnicas (CONICET). Docente de la Facultad de Ciencias Económicas (UBA).

Correo electrónico: pilarpique@ gmail.com

${ }^{3}$ Lic. En Economía (UBA). Doctorando en Economía (UBA).

Correo electrónico: lean.navarro@ gmail.com

${ }^{4}$ Lic. En Economía (UBA). Master Analyse Politique et Économie (Université Paris Sorbonne). Doctorando en Economía (UBA).

Correo electrónico: martinharraca@ gmail.com

${ }^{5}$ Lic. En Economía (UBA). Doctorando en Economía (UBA). Becario doctoral de Consejo Nacional de Investigaciones Científicas y Técnicas (CONICET). Docente de la Facultad de Ciencias Económicas (UBA) y de la Universidad Nacional de La Matanza (UNLaM)

Correo electrónico: pablobenchimol@gmail.com

${ }^{6}$ Lic. En Economía (UBA). Magister en Historia del Arte Argentino y Latinoamericano, Universidad Nacional de San Martín, Provincia de Buenos Aires, Argentina. Analista económico en la Comisión Nacional de Defensa de la Competencia. Docente de la Facultad de Ciencias Económica (UBA). Correo electrónico: cataldama@gmail. com

Para citar este artículo use: Piqué P., Navarro, L., Harracá, M., Benchimol, P., \& Aldama, C. (2017). Investigación sobre la transición entre las doctrinas mercantilistas y el nacimiento de la Economía Política. Un análisis de los aportes de Thomas Mun, James Steuart y David Hume. Civilizar Ciencias Sociales y Humanas, 17(32), 119-132. Doi: $10.22518 / 16578953.822$ 


\section{Introducción}

En el presente trabajo nos proponemos estudiar la progresión del pensamiento económico de los siglos XVII y XVIII, a partir del análisis de obras originales emblemáticas del período como las de Thomas Mun, James Steuart y David Hume. Debe tenerse presente que la motivación para este escrito se origina en un proyecto de mayor alcance de este equipo de investigación, que se propuso escribir un conjunto de artículos que pudieran dar cuenta de la discusión de la necesidad del estudio de la historia del pensamiento económico para el desarrollo de la ciencia económica hoy. En el primero de estos artículos (Aldama, Benchimol, Harracá, Navarro \& Piqué, 2012) nos propusimos revalorizar la importancia de la doctrina escolástica medieval en la formación de la ciencia económica moderna. Así, dedicamos particular atención al pensamiento económico de los siglos XIV y XV, y la ruptura que implicó respecto de la concepción del mundo medieval, dominada por la tradición aristotélico-tomista. En dicho artículo la investigación tuvo como principal foco el proceso por medio del cual el estudio del comercio y de los fenómenos monetarios del incipiente capitalismo comercial llevó a las primeras nociones modernas acerca de la mercancía, del dinero y del capital, que fueron luego desarrolladas por los mercantilistas.

El presente trabajo puede leerse como una continuación natural de aquel. Aquí nos centraremos en mostrar cómo las concepciones elaboradas por las primeras doctrinas liberales de los siglos XVII y XVIII sobre los procesos económicos comenzaron a revelar el carácter fragmentario y limitado de las concepciones de la doctrina mercantilista ante la emergencia y desarrollo del capitalismo industrial. Asimismo, indagaremos respecto a cómo estas doctrinas se esforzaron progresivamente por entender a los procesos económicos en un contexto universal y no unilateralmente nacional, pugnando por afianzar la conformación de un cuerpo teórico unificado del sistema capitalista como un todo, lo que luego daría nacimiento a la Economía Política.

El trabajo se estructurará en cinco partes. En la primera de ellas reseñaremos las principales contribuciones de la literatura existente acerca del nacimiento de la Economía Política y el ocaso de las ideas mercantilistas y expondremos, en ese marco, qué contribución representa el presente artículo para aquella literatura. En la segunda parte esbozaremos los límites de las explicaciones doctrinarias de fines del siglo XVII y comienzos del XVIII ceñidas al ámbito de la circulación mercantil, a partir del análisis de la obra de Thomas Mun. En la tercera parte, estudiaremos los "arranques en falso" de una teoría general $^{1}$ de la reproducción capitalista ${ }^{2}$ a partir de la reconstrucción de los principales aportes de los escritos de James Steuart. En la cuarta, discutiremos el contenido de la crítica de Hume a las nociones mercantilistas de la balanza comercial, así como el terreno que prepara para la gestación de la Economía Política. En la quinta expondremos las conclusiones principales del trabajo.

\section{EI nacimiento de la Economía Política y el ocaso de las ideas mercantilistas}

\section{Contribuciones de la literatura existente.}

Las doctrinas económicas desarrolladas en Inglaterra y Escocia hacia finales del siglo XVII y comienzos del siglo XVIII han sido catalogadas por la literatura especializada en Historia del Pensamiento como una "reacción contra el mercantilismo" (Medema \& Samuels, 2004; Mills, 2002), como "antecesoras o precursoras de la Economía Política Clásica" (Colander \& Landreth, 1994; Screpanti \& Zamagni, 2005), como "el amanecer del pensamiento económico moderno" (Haney, 1936), como expresiones de la "continuidad y el cambio entre las doctrinas mercantilistas y las clásicas" (Magnusson, 2015), o como los portavoces de la "transición hacia la economía clásica" (Rima, 2003), entre otros. 
La atención sobre su carácter transicional se posó sobre la transformación de las premisas económicas empíricas que eran el sostén de las doctrinas mercantilistas. La difusión del comercio tendió a reducir los diferenciales de precios entre las regiones y las naciones, lo que causó una merma en los márgenes de ganancias y un debilitamiento de las grandes compañías monopólicas (Romero, 1987). Los gremios y los maestros artesanos devinieron simples trabajadores asalariados al compás de la creación de una clase burguesa que no surgía del comercio y cuyos intereses entraban en conflicto con el de los comerciantes (Schumpeter, 1954), cuadro que contribuyó a instalar la percepción de que los precios y las ganancias no eran un simple reflejo de las condiciones de la demanda. Como señala Roll (1969), se tornó cada vez más difícil sostener que la riqueza nacía del cambio, y que el valor de cambio (expresión de la riqueza) y la ganancia (por medio de la cual se acrecienta la riqueza) surgían del comercio. Sobre esta base surge, entonces, la necesidad de reformular los conceptos de valor y de riqueza.

La noción de que el origen del beneficio no se encontraba en la esfera del intercambio era, a la vez que una nueva premisa del pensamiento económico, un argumento a favor de la legitimación de esa naciente clase burguesa incipientemente industrial, quien progresivamente se encontraba en condiciones de exigir mayor libertad de comercio y mayor "autonomía" en los "asuntos económicos" (Rubin, 1979). El interés de esta nueva clase fue, desde el comienzo, opuesto al de los antiguos mercaderes (Hunt, 2015) y sus exigencias tendrían el aval de los pensadores económicos de su tiempo, quienes no serían tampoco eminentemente mercaderes como lo fueron en los siglos anteriores, sino personajes vinculados a las nacientes industrias o incluso filósofos de gran renombre, que ampliarían la perspectiva y el campo de interés del pensamiento económico (Mills, 2002). La flamante actitud era crecientemente liberal: se entendía que una mayor libertad en aquellos asuntos sería beneficiosa para la sociedad en su conjunto (Rima, 2003).

El trabajo que aquí proponemos no reniega de estas valiosas interpretaciones que se han hecho sobre las doctrinas económicas de finales del siglo XVII y comienzos del siglo XVIII. Lo que sí se propone es complementarlas a partir de instalar como eje de discusión lo que llamaremos las "transiciones del concepto", que aquí presentamos como el paso de la conformación de una teoría general de la circulación mercantil a la conformación de una teoría general sobre el proceso de reproducción capitalista. En la historia de las doctrinas económicas, los momentos de transición entre una teoría general y otra aparecen como episodios de gran efervescencia y producción intelectual, signados por el choque entre expresiones doctrinarias que se sostienen tanto sobre la teoría general que muestra señales de agotamiento como sobre la nueva, que está aún en trabajo de parto. En esta coyuntura se suceden también todo tipo de criaturas intelectuales, portavoces de un pensamiento híbrido que ilustra la convulsión reinante, $\mathrm{y}$ que ensayan arranques teóricos en falso, que no alcanzan a cristalizarse en una clara superación de la teoría general previa, pero que tampoco son en vano, sino que preparan el terreno a una posterior revolución. Estas revoluciones en el pensamiento se completan, finalmente, con exponentes descollantes, que encarnan decididamente el espíritu de la época, sintetizando del pensamiento precedente los aspectos teóricos fundamentales y legando problemas teóricos que las siguientes generaciones deberán comprender y resolver.

\section{Desarrollo del alcance de las contri- buciones de nociones transicionales mediante el concepto de pensa- miento híbrido.}

En la "Cuestión I" abordamos una primera transición, no dentro del marco de la Economía Política, sino aquella que dio origen 
al pensamiento moderno, en general, y en particular, la que marcó la génesis de la Ciencia Económica Moderna, la cual se corporizó en el nacimiento de su primera teoría general: la teoría general del intercambio mercantil (Aldama et al., 2012). En el trabajo actual nos centramos ya en el período histórico que presenta la tensión entre un pensamiento que se encuentra atravesado por expresiones de agotamiento de aquella teoría (por momentos muy vívidas en las sucesivas críticas al pensamiento mercantilista), y el nacimiento de una incipiente teoría general de la reproducción capitalista, que empieza a construirse sobre la base de las nociones de la teoría general anterior y sobre nuevas nociones que surgen de la vida empírica del incipiente capitalismo industrial.

En el siglo XVIII, período de gran creación conceptual, se ubica la transición que atraviesa el propio objeto de estudio que nos compete: el sistema capitalista en su transformación desde su inicio decididamente comercial a su evolución industrial. En este marco la Economía Política (como parte del cuerpo general de la ciencia de la época) se propuso comprender las leyes que rigen el movimiento (y, por tanto, el equilibrio) del sistema de reproducción capitalista (Cassirer, 1984; Danford, 1990; Griswold, 1999). En este sentido, los autores elegidos representan esta transición, al encarnar en sus escritos el pensamiento híbrido a partir del cual construyen sus explicaciones sobre los fenómenos económicos. Sin poder terminar de despojarse de las primeras nociones de la mercancía, el dinero y el capital referidas a la circulación mercantil, los autores de la transición a la Economía Política, comenzarán a preguntarse por nociones propias del momento de transformación técnico material y del proceso de reproducción capitalista en su conjunto. Al hacerlo, lo incorporarán al objeto de estudio (cuyas determinaciones, hasta el momento, únicamente encontraban sentido en la circulación mercantil) ampliándolo al sistema de reproducción social del sistema capitalista, sin aún formular (ni por tanto desarrollar) acabadamente las leyes de su funcionamiento (Levín, 1999).

Las señales de agotamiento de un pensamiento económico reducido a la esfera del intercambio mercantil se verán, entonces, en las primeras críticas de las doctrinas liberales a las políticas proteccionistas y a las estrategias económicas que propugnaron los primeros mercantilistas. Luego se expresarán en las preguntas de la época respecto de la instancia de creación material de las mercancías que se comercian, del rol del comerciante en el marco de la producción, interrogantes sobre la población -entendida como mano de obra-, su salud, su reproducción y su educación, y sobre las determinaciones de los precios y la ganancia.

\section{Primeras críticas a la doctrina mercantilista}

Aún sin poder desprenderse de las nociones establecidas durante los siglos XV y XVI respecto de la mercancía, el dinero y el capital, a comienzos de siglo XVII la doctrina mercantilista empieza a mostrar ciertas grietas, y sus recomendaciones de política sufren las primeras críticas $^{3}$. Para dar un comienzo, coincidente en lo cronológico y en lo conceptual, encontramos el texto de Thomas Mun (1571-1641) -quien se desempeñó como director de la Compañía de Indias, empresa de origen británico que mantuvo el monopolio comercial de Europa Occidental con las "Indias Orientales"- titulado $L a$ riqueza de Inglaterra por el comercio exterior. Este escrito fue publicado póstumamente en 1664, y es el que con mayor claridad logra dar un nuevo sentido a los problemas planteados por el primer mercantilismo ${ }^{4}$.

La crítica de Mun representa un quiebre, en tanto es el primero en explicar al comercio como un ciclo de compra-venta constante, entendiendo a la balanza comercial como un reflejo de este flujo. Plantea una concepción de riqueza ligada a la acumulación de capital, comprendido como una acumulación de dinero 
transformado en ganancia (en tanto resultado de la actividad comercial) y no como un mero acopio de metales preciosos.

Su crítica, además, supone una inversión en los términos de la relación existente entre el resultado de la Balanza Comercial y el tipo de cambio: no será para Mun la apreciación del tipo de cambio lo que genere una mayor entrada de metálico, sino que será una mayor entrada de metálico, como reflejo de un resultado comercial superavitario, lo que origine una apreciación del tipo de cambio. O, lo que es lo mismo: "No es la devaluación de nuestro dinero en el cambio sino el que nuestro comercio sea superado lo que origina la salida de nuestro dinero" (Mun, 1664, p. 22).

En consonancia con estos postulados, Mun propone que en la relación comercial de la nación con otros Estados no debe perseguirse un resultado superavitario con cada uno de ellos, sino un superávit de la Balanza Comercial General. En este sentido, aboga por un proteccionismo más refinado en el que no se encuentren completamente vedadas todas las importaciones, mas solo aquellas que sean producidas al interior del reino. En definitiva, propone una política que considere la naturaleza del proceso de acumulación del capital y generación de riqueza de la nación (como la importación de mercancías de las "Indias Orientales" para su reexportación a los mercados europeos). Bajo esta luz, las políticas proteccionistas precedentes comienzan a verse como restricciones al desarrollo del comercio y a la generación de la riqueza de los reinos, y no como estrategias para su impulso.

Mun logra dar cuenta de la relación que mantiene el comercio con el aumento de la riqueza del reino, lo cual supone una noción de riqueza distinta a la que presentaban los primeros mercantilistas 5 . Para este autor es únicamente el dinero que ingresa al reino como resultado del comercio -el capital en tanto acumulación de ganancia- el que contribuye a incrementar su riqueza. En este sentido, las políticas proteccionistas destinadas a acaparar metales preciosos y la adulteración de la moneda local son, para Mun, estrategias estériles (o incluso contraproducentes) que no devendrán en un aumento de la riqueza. La exportación de metales preciosos, por su parte, no genera una merma en la riqueza del reino al ser concebida como parte del flujo de la actividad comercial. En palabras del autor:

Debemos considerar que todos los medios y recursos que (en el proceso comercial) compelen a la riqueza a entrar al reino, no la hacen nuestra por esta razón, pues esto puede lograrse solamente por una ganancia legítima y ésta de ninguna manera puede alcanzarse sino por el excedente de nuestro comercio y este excedente disminuye con las restricciones; de consiguiente tales restricciones positivamente impiden el incremento de nuestra riqueza (Mun, 1664, p. 20).

Asimismo, dando cuenta de las contradicciones existentes entre la realidad práctica de los primeros mercantilistas y el funcionamiento del sistema capitalista y las políticas y estrategias económicas aún vigentes, Mun alerta sobre la inconveniencia de la abundancia de dinero en la Nación ya que encarece los artículos domésticos reduciéndose su demanda. Esta crítica viene a reforzar la nueva noción de riqueza que postula Mun: la mera acumulación de dinero es estéril o incluso perjudicial para enriquecer a la Nación.

No es el conservar nuestro dinero en el reino, sino la necesidad y empleo de nuestras mercancías en los países extranjeros, y nuestra necesidad de sus productos lo que origina su salida y consumo en todas partes y lo que hace un rápido y extenso comercio (Mun,1664, p. 9).

Sin embargo, el comercio sigue siendo para este autor el motor de la riqueza, puesto que es allí donde el dinero, en tanto equivalente general, deviene capital. Sin quizás comprender acabadamente las determinaciones de estas dos categorías, Mun atisba a ver sus diferencias apartándose del pensamiento mercantilista inicial. 
El texto de Mun anticipa en casi cien años la crítica que luego hará Hume y da cuenta del proceso de transformación del propio objeto de estudio hacia un sistema capitalista con un comercio internacional cada vez más articulado. En este sentido, Mun recomienda vender caro a los Estados vecinos aquellas mercancías con una demanda poco elástica en relación con el precio, y vender barato aquellas para la cuales existe competencia de otros Estados y cuya demanda posee una mayor reacción a variaciones en el precio ${ }^{6}$.

La propuesta de este autor deja entrever que la difusión del comercio ha servido para una expansión de la producción y difusión de las técnicas productivas. La especialización productiva y la reducida "elasticidad de la demanda" que antes definía el funcionamiento comercial entre los Estados-naciones comienzan a diluirse ante el despliegue de la mercancía como relación social general y la consolidación de un mercado mundial (Roncaglia, 2006). La competencia por precio aparece como primer síntoma de esta universalización que impone la relación mercantil en el vínculo entre Estados-naciones.

A pesar de la perspectiva crítica que posee Mun respecto de la primera doctrina mercantilista aún vigente, sus desarrollos no dejan de ubicarse en el terreno de la circulación mercantil, en tanto sus nociones sobre los precios, el dinero y el capital no escapan de la esfera del mercado. Así, la escasez o abundancia de dinero es lo que determina para Mun el tipo de cambio, y es este el que determina a su vez los precios de los artículos y del propio dinero (el precio del dinero entendido como interés). En este sentido, los precios y el interés no se determinan fuera del ámbito de la circulación mercantil. La ganancia, por su parte, es comprendida por este autor como el excedente originado como producto del comercio, por lo que el origen del capital tampoco se da por fuera del momento social de la producción, presentándose como una diferencia casi contingente entre el precio de venta y el de compra de las mercancías.

\section{Primeros interrogantes sobre el "más allá" de la esfera de circulación mercantil}

Prácticamente de forma contemporánea a Mun, se presentan una serie de pensadores -Richard Cantillon, William Petty y James Steuart- que mostrarán un mayor interés por el momento de transformación material, hasta aquí parcialmente desdeñado, que se traducirá, entre otras cosas, en diferentes explicaciones acerca de la determinación de los precios. Sin embargo, este abordaje no logrará articularse como ley general del valor. Se tratará de pasos en falso en la formulación de la teoría del valor que quedarán en un momento de maduración intermedio, dejando el camino allanado para que la Economía Política acometa con este desafío de manera deliberada.

En este nuevo contexto de pensamiento, las discusiones acerca de la determinación de los precios presentarán también elementos relevantes que, en perspectiva, permiten comprender el trayecto hacia la elaboración que luego emprenderá Adam Smith.Tomaremos aquí particularmente el análisis de la obra de James Steuart. Su indagación sobre la determinación de los precios pondrá el foco en el momento de transformación material de la producción y, sin embargo, veremos cómo queda a mitad de camino en la formulación de una ley del valor. Resultará también ilustrativa la manera en que, sobre la base de un concepto de valor sin suficiente maduración, dejará también incompletos sus importantes avances en el despliegue del concepto de ganancia.

Steuart (1767) expone un desarrollo en $A n$ inquiry into the principles of Political Economy ciertamente ambicioso y con una perspectiva que abarca una gran variedad de problemas de la vida social en la que se desenvuelve, tal como se anticipa en el título completo de la obra ${ }^{7}$. Salta a la vista en el propio título, la incorporación del rótulo "Economía Política". Esta aparición exigirá, asimismo, una discusión 
en torno al objeto de la disciplina que, por un lado, apunta a trascender al simple intercambio mercantil, incorporando al momento de transformación material y que, por otro lado, pretenderá tener un alcance sistémico, aunque entendiendo el sistema únicamente como la economía nacional:

Economy, in general, is the art of providing for all the wants of a family, with prudence and frugality. What economy is in a family, political oeconomy is in a state: with these essential differences, however, that in a state there are no servants, all are children: that a family may be formed when and how a man pleases, and he may there establish what plan of oeconomy he thinks fit; but states are found formed, and the Oeconomy of these depends upon a thousand circumstances. The statesman (this is a general term to signify the legislature and supreme power, according to the form of government) is neither master to establish what oeconomy he pleases, or, in the exercise of his sublime authority, to overturn at will the established laws of it, let him be the most despotic monarch upon earth (p. 2).

Sobre esta base, Steuart (1767) presentará una concepción sobre la determinación del real value de las mercancías y del beneficio, introducido inicialmente como profit upon alienation. En efecto, respecto del valor de las mercancías, Steuart sostiene que se fijará a través de tres elementos. En primer lugar, se deberá tener en cuenta el tiempo de trabajo necesario para obtener la mercancía, suponiendo que no se trata de un tipo de trabajo que posee particulares ventajas ni desventajas respecto de la media:

The first thing to be known of any manufacture when it comes to be sold, is, how much of it a person can perform in a day, a week, a month, according to the nature of the work, which may require more or less time to bring it to perfection. In making such estimates, regard is to be had to what, upon an average only, a workman of the country in general may perform, without supposing him the best or the worst in his profession; or having any peculiar advantage or disadvantage as to the place where he works (p. 11).

En segundo lugar, se debe considerar el valor de la subsistencia del trabajador y los gastos necesarios, tanto para sus necesidades personales como para los instrumentos pertenecientes a su profesión. En palabras de Steuart (1767): "The second thing to be known, is the value of the workman's subsistence and necessary expense, both for supplying his personal wants, and providing the instruments belonging to his profession, which must be taken upon an average as above" (p. 11).

En tercer y último lugar, deberá considerarse el "valor de los materiales" para la manufactura de los productos: "The third and last thing to be known, is the value of the materials, that is the first matter employed by the workman" (p. 12).

En definitiva, lo que explica el "valor" en los términos de Steuart es el tiempo de trabajo necesario, pero también los valores de los materiales requeridos y el valor de la subsistencia de los trabajadores involucrados en el proceso productivo. Es decir, a pesar de introducir al tiempo de trabajo como una variable determinante, se completa su explicación del valor a partir de otros valores, lo cual da lugar a una explicación ciertamente circular. Por lo tanto, a pesar de mostrar un interés y un abordaje que se adentra en el proceso de transformación material, finalmente, el principio de valor no queda suficientemente maduro y lleva a Steuart a caer en un razonamiento con el que, en definitiva, no logra trascender la esfera de la circulación.

Queda, sin embargo, un segundo elemento por considerar en la doctrina de Steuart sobre la determinación de los precios: la ganancia. Steuart ofrece una clasificación en dos tipos de ganancias (o pérdidas), la ganancia positiva y la ganancia relativa.

De un lado, las ganancias relativas serán aquellas que implican una pérdida para 
alguien y que no agregan nada al stock general de ganancias: "Relative profit, is what implies a loss to somebody, it marks a vibration of the balance of wealthbetweenparties, butimplies no addition to the general stock"(Steuart, 1767, p. 23). Se trata aquí de una redistribución de la ganancia entre distintos individuos, sin que pueda contenerse una ganancia a nivel agregado (sistémico): el consabido "unos ganan lo que otros pierden", o "juego de suma cero".

Del otro lado, las ganancias positivas implican la creación de una ganancia a nivel general. Sin embargo, a la hora de dar cuenta de este proceso, sobre la base de un incompleto principio de valor, Steuart "deja pasar la oportunidad" (Rubin, 1979) de exponer el origen efectivo de este tipo de ganancia y vuelve recurrentemente al profit upon alienation.

Precisamente, esta determinación resulta fundamental para comprender un sistema de reproducción fundado en un régimen industrial más maduro, que tiene como eje la acumulación de capital a nivel general y no simplemente una redistribución de ganancias entre sus distintos capitales. Es aquí donde se presenta de manera vívida el límite de la teoría general de la circulación mercantil que, a través de su comprensión del valor primigenia, no puede echar luz sobre la comprensión sistémica de la ganancia. Los intentos de Steuart por dar cuenta de la determinación del valor, y el corolario respecto de la noción de excedente social, así lo demuestran.

\section{David Hume: en los lindes de la Economía Política}

Si se trata de autores que logran dar cuenta de la necesidad de un campo de estudio más amplio, David Hume es, sin dudas, uno de los más importantes. Contemporáneo de Adam Smith, Hume es uno de los autores más influyentes de la Ilustración. Con su Tratado sobre la naturaleza humana, que atraviesa la totalidad de las disciplinas científicas, Hume logra involucrarse tanto en discusiones sobre el conoci- miento, la moral y las pasiones humanas, como en el demoledor análisis de la razón que se basa en la imposibilidad de justificar racional y empíricamente la relación causa-efecto ${ }^{9}$. Hume en este sentido es un claro exponente Ilustrado (y escéptico) del Siglo de las Luces, que ha logrado mostrar en el contexto de los siglos XVII y XVIII, una serie de discusiones en el ámbito de la sociedad civil, los cuales han encontrado su cauce y una forma más acabada en la Economía Política (Broadie, 2003; Groenewegen, 2002; Norton, 1995).

El golpe que Hume, en sus Ensayos Políticos, le propina al mercantilismo es demoledor y prácticamente definitivo: contrario a lo que solía postular esta doctrina, Hume invierte la relación entre cantidad de dinero y riqueza, al sostener que la abundancia de dinero no es sinónimo de prosperidad para la nación, ni su escasez causa de malestar generalizado ${ }^{10}$. El dinero es un instrumento que sirve para facilitar el intercambio de mercancías, pero no es su simple incremento lo que permite sentar las bases de la prosperidad:

Money is not, properly speaking, one of the subjects of commerce; but only the instrument which men have agreed upon to facilitate the exchange of one commodity for another. It is none of the wheels of trade: It is the oil which renders the motion of the wheels more smooth and easy. If we consider any one kingdom by itself, it is evident, that the greater or less plenty of money is of no consequence; since the prices of commodities are always proportioned to the plenty of money (Hume, 1752a, párr. 1) ${ }^{11}$.

Hume encuentra que en los países donde el dinero es muy escaso, el comercio languidece y el Estado no puede recaudar ningún impuesto. Por tanto, la pregunta que se plantea es ¿cómo conciliar estos hechos con el principio de que la cantidad de oro y plata es en sí misma indiferente en este respecto?

La respuesta es que se trata, en rigor, de una contradicción aparente: la escasez de dine- 
ro y la debilidad de una nación no son más que efectos colaterales de "las costumbres y usos de los pueblos". Si bien pueden presentarse juntos ambos efectos, obedecen a una circunstancia de orden superior. Justamente, Hume sostiene que las sociedades, al engrandecer su industria, permiten una distribución generalizada de oro y plata, lo cual impide que se concentre en pocas manos y se obstaculice el florecimiento de la nación. El foco de Hume (1752b) hacia el despliegue de la industria, un Estado bien poblado y cultivado, vuelve a mostrar la relevancia que le asigna a las determinaciones propias de la transformación material, aunque se presenten de manera fragmentaria y no sistematizada:

Now, it is evident, that the same causes, which would correct these exorbitant inequalities, were they to happen miraculously, must prevent their happening in the common course of nature, and must for ever, in all neighbouring nations, preserve money nearly proportionable to the art and industry of each nation (párr. 11).

Así, será el despliegue de la industria lo que explicará la cantidad de dinero que circulará en la nación, y no esta última la que explicará la prosperidad del pueblo. Esto lo vemos en el correlato entre las nociones sobre el dinero y las políticas comerciales necesarias para el progreso de una nación. Aquí Hume vuelve a mostrar los límites de las prescripciones emblemáticas de los mercantilistas. En este caso, lo que postula es la ineficacia o esterilidad en el largo plazo de aplicar obstáculos a la salida de oro y plata de la nación. El argumento es el siguiente: a partir de la teoría cuantitativa de la moneda, un incremento de las cantidades dinerarias en circulación (gracias a la política comercial proteccionista) traería como consecuencia una alza proporcional de los precios a nivel nacional. Sin embargo, este aumento de los precios internos traería aparejada una pérdida de "competitividad" de la producción local y, en definitiva, terminaría achicando el ingreso de oro y plata por la vía del comercio internacional ${ }^{12}$. A este proceso de ajuste se le denominaría posteriormente el mecanismo precio-flujo (Screpanti \& Zamagni, 2005). La conclusión para el largo plazo, entonces, es que las políticas proteccionistas, que buscaban acumular una mayor cuantía de oro y plata al interior del país, terminarían siendo fugaces e ineficaces ${ }^{13}$. Finalmente, el nivel de dinero en circulación en la nación terminaría volviendo a su nivel inicial, determinado por la capacidad industrial de la nación.

La contradicción que surge es que, luego de "abrir la economía", la teoría cuantitativa expuesta por los mercantilistas para una "economía cerrada" no rige de igual forma. En el marco de una economía cerrada, un incremento de dinero traería aparejado una suba proporcional de los precios. Aquí, la cantidad de dinero se presenta como una variable independiente que puede subir o bajar y que luego simplemente impacta en los precios (dinero $=>$ precios). En una economía abierta, en cambio, lo que se da es la imposibilidad de modificar de manera estructural el nivel de dinero al interior de la nación, ya que todo movimiento por fuera del equilibrio sería ajustado a largo plazo a través del mecanismo precio-flujo. La relación causal una vez que se considera a la economía abierta, donde aparece en forma evidente la conformación de un mercado de carácter universal, se invierte respecto a la postulada por la teoría cuantitativa de la moneda vigente en la economía cerrada. Esta nueva versión expuesta por Hume sintetiza el quiebre respecto de la discusión en torno a la política comercial y a la noción de dinero dominante en el mercantilismo, llevando hasta sus consecuencias últimas el pensamiento mercantilista y mostrando la esterilidad de sus postulados políticos.

Sin embargo, aunque su escrito represente un triunfo teórico y político contra la doctrina mercantilista, su análisis no tuvo igual claridad a la hora de exponer la fuente de la determinación de los precios no ceñida unilateralmente a la circulación mercantil. Si bien su genio allana el camino teórico, ese desarrollo será profundi- 
zado por la Economía Política de fines del siglo XVIII y comienzos del XIX, especialmente de Adam Smith y de David Ricardo.

\section{Conclusiones}

En el presente trabajo nos propusimos estudiar la progresión del pensamiento económico de los siglos XVII y XVIII, a partir del análisis de obras originales emblemáticas del período como las de Thomas Mun, James Steuart y David Hume. Para ello, en primer lugar, reseñamos las contribuciones de la literatura existente sobre el tema y propusimos nuestro propio abordaje interpretativo, centrado en la idea de un "pensamiento híbrido" que se encuentra tensionado por expresiones de agotamiento de la teoría general de la circulación mercantil (por momentos muy vívidas en las sucesivas críticas al pensamiento mercantilista), y el nacimiento de una incipiente teoría general de la reproducción capitalista, que empieza a construirse sobre la base de las nociones de la teoría anterior y sobre nuevas nociones que surgen de la vida empírica del naciente capitalismo industrial. En la segunda parte reconstruimos los rasgos del pensamiento híbrido en la obra de Mun, quien, si bien rechaza la noción de riqueza como mero acopio de metales preciosos (lo que se traduce en una crítica a las recomendaciones de política de los primeros mercantilistas), proclama, en cambio, que la riqueza es fruto de la actividad comercial, sin tener en cuenta qué papel juega el proceso de transformación técnico material en la producción de valor y de riqueza.

En la tercera parte, desarrollamos las tensiones del pensamiento de James Steuart, quien empieza a mostrar que tanto el valor como la ganancia no surgen exclusivamente del ámbito de circulación mercantil. En el caso del valor, señaló que había que tener en cuenta como uno de sus determinantes al tiempo necesario de trabajo para producir las mercancías, aunque sostuvo que este no era el único factor implicado y que había que tener en cuenta otros precios de mercado (como los costos para emplear al trabajador y los costos de los materiales). En el caso de la ganancia, Steuart señaló que la idea de que "unos ganan lo que otros pierden" (que él llamó ganancia relativa) no sirve para comprender la creación de ganancia positiva (es decir la creación de ganancia a nivel general del sistema), aunque no llegó a dar pistas acerca de cuáles son los determinantes de esa ganancia general.

En la cuarta parte, reexploramos la obra de David Hume para mostrar cómo su valiosa crítica a las recomendaciones de política mercantilista se basó en llevar algunas premisas de la circulación mercantil hasta sus últimas consecuencias, pero no en desarrollar una teoría general más completa de la reproducción mercantil. Su aporte consistió en dejar de pensar en términos de "economías cerradas" y apuntar a comprender la dinámica del sistema capitalista en su conjunto, base sobre la que se gestaría la Economía Política.

\section{Notas}

1 Empleamos el sintagma "teoría general" para referirnos a teorías sobre el sistema capitalista en su conjunto.

2 Concebimos al proceso de reproducción capitalista como la unidad de dos momentos: el momento de transformación técnico material (o natural) y el momento social (o de circulación mercantil o del mercado). El momento natural comprende un proceso de transformación técnico material, mediado por el trabajo humano, el cual es aquí una fuerza natural junto con otras fuerzas naturales; el momento social se concreta en la relación de ese carácter en la que los productos del trabajo se transforman en producto social (Levín, 2003, p. 1). A lo largo del trabajo nos referiremos a autores que se centraron en uno o en otro de esos dos momentos. Llamaremos teoría general de la circulación mercantil al primer momento y teoría general de la reproducción capitalista al segundo. 
3 A pesar de que la doctrina mercantilista no se apoya sobre un cuerpo de obra coherente y acotadosino más bien sobre los escritos de numerosos autores de distintas naciones de Europa Occidental que escribieron sobre problemáticas económicas durante los siglos XVI, XVII y parte del XVIII, se pueden hallar en estos textos ciertas nociones en común sobre la mercancía, el dinero, el capital y, sobretodo, un fuerte consenso respecto a beneficios de mantener una política de comercio exterior de carácter proteccionista. Existe numerosa literatura especializada que se ha dedicado a debatir acerca de la pertinencia de englobar tan diversos autores bajo el rótulo de "mercantilistas", sin embargo, dos de los núcleos de ideas económicas más importantes que confrontaron directamente con las nociones del pensamiento mercantilista, los fisiócratas en Francia y Adam Smith en Escocia, han utilizado libremente esta clasificación para identificar aquellos conceptos económicos y propuestas de política a las que se oponían. Para más detalles sobre esta temática se pueden ver: Rubin,1979; Magnusson, 2015; Screpanti \& Zamagni, 2005, entre otros.

4 También cabe mencionar Discourses upon trade, un texto del comerciante inglés Dudley North que fue publicado en 1691 y que continua indagando sobre las problemáticas que ya había señalado Thomas Mun anteriormente.

5 Rubin (1979) traza una distinción entre la noción de riqueza de los primeros mercantilistas y los mercantilistas tardíos, entre los cuales se encuentra Mun. Al respecto dice: "The view is often expressed that mercantilist doctrine is reducible to the declaration that precious metals are the sole form of wealth. Adam Smith sharply criticizes «the absurd notion of the mercantilists that wealth consists of money». And yet such characterization is quite unjust. They looked upon increases in the quantity of precious metals not as a source of a nation's wealth, but as one of the signs that this wealth was growing. It is only the early mercantilists whose intellectual horizons remained naively confined to the sphere of monetary circulation. The late mercantilists, in putting forth the doctrine of the «balance of trade» uncovered the connection between the movement of the precious metals and the overall development of trade and industry" (pp. 40-41).

${ }^{6}$ Al respecto Mun (1664) dice: "En nuestras exportaciones no solamente debemos atender a nuestros sobrantes, sino también debemos tomar en consideración las necesidades de nuestros vecinos, por lo que se refiere a los efectos que no quieran recibir o de que no puedan ser provistos de ninguna otra parte; así estaremos en posibilidad (además de dar salida a nuestras materias) de ganar otro tanto por su manufactura, puesto que podemos y también debemos venderlas caras, hasta tanto que el precio alto no ocasione una menor salida en cantidad. Pero el sobrante de nuestras mercancías que los extranjeros usan y que también puedan obtener de otras naciones, con pocos inconvenientes, puede reducir su salida por el uso de mercancías de igual clase de otros lugares; en este caso debemos esforzarnos por vender tan barato como nos sea posible, mejor que perder el mercado de tales efectos, ya que hemos encontrado, por la buena experiencia de los últimos años, que estando en posibilidad de vender nuestras telas baratas en Turquía, hemos aumentado grandemente su salida, y los venecianos han perdido mucho en su mercado de las suyas en esos países, porque son más caras" (p.4).

7 El título completo de la obra de James Steuart es, en efecto, "An Inquiry into the Principles of Political Economy: being an Essay on the Science of Domestic Policy in Free Nations. In which are particularly considered Population, Agriculture, Trade, Industry, Coin, Interest, Circulation, Banks, Exchange, Public credit, and Taxes" (Steuart, 1767, p. 1).

${ }^{8}$ De hecho, es la primera obra en habla inglesa que ostenta el sintagma "Economía Política" en su título. 
9 En este sentido, demuestra que no existe validez lógica en esperar que en el futuro se repitan los sucesos que se encontraban vinculados en el pasado. Sin embargo, el hábito y la costumbre han guiado al hombre para desenvolverse cotidianamente y organizar $\mathrm{su}$ accionar inmediato a través de estas recopilaciones de hechos del tipo causa-efecto. Por tanto, pese a su invalidez lógica, este cúmulo de experiencias servirá como una especie de guía "práctica operativa" (Cassirer, 1984).

10 "Where coin is in greater plenty; as a greater quantity of it is required to represent the same quantity of goods; it can have no effect, either good or bad, taking a nation within itself; any more than it would make an alteration on a merchant's books, if, instead of the ARABIAN method of notation, which requires few characters, he should make use of the ROMAN, which requires a great many"(Hume, 1907, p. 3).

${ }^{11}$ En rigor, Hume aceptará que a corto plazo, un shock monetario positivo puede tener "efectos reales", hasta que los precios vuelvan a ajustarse al alza. Solo en este lapso entre el shock inicial y el nuevo nivel de precios, podrán registrarse efectos positivos sobre la actividad económica. El efecto a largo plazo será neutral (Screpanti \& Zamagni, 2005).

${ }^{12}$ En este razonamiento de Hume debe tenerse en cuenta que, como dijimos anteriormente, a diferencia de lo que suponían los mercantilistas, para que pueda llevarse a cabo el ajuste descrito, tienen que existir "elasticidades-precio" de las exportaciones relativamente altas. En este sentido, puede suponerse que en épocas anteriores a Hume, donde los monopolios comerciales tenían una influencia mayor, era más difícil pensar en la existencia de un ajuste de este tipo (Rubin, 1979).

13 "To account, then, for this phenomenon, we must consider, that though the high price of commodities be a necessary consequence of the encrease of gold and silver, yet it follows not immediately upon that encrease; but some time is required before the money circulates through the whole state, and makes its effect be felt on all ranks of people. At first, no alteration is perceived; by degrees the price rises, first of one commodity, then of another; till the whole at last reaches a just proportion with the new quantity of specie which is in the kingdom. In my opinion, it is only in this interval or intermediate situation, between the acquisition of money and rise of prices, that the encreasing quantity of gold and silver is favourable to industry" (Hume, 1752a, párr. 7).

\section{Referencias}

Aldama, C., Benchimol, P., Harracá, M., Navarro, L., \& Piqué, P. (Agosto, 2011). La necesidad del estudio de la historia del pensamiento económico. Cuestión I. Presentado en la IV Jornadas de Economía Crítica (JEC) Dilemas de acción y del pensamientocrítico latinoamericano: desarrollo, Estado, movimientos sociales, Ciudad de Córdoba, Argentina.

Aldama, C., Benchimol, P., Harracá, M., Navarro, L., \& Piqué, P. (2012). Las transformaciones de las representaciones del mundo desde el tránsito de la Edad Media al Capitalismo Comercial. Revista Nueva Economía, 19(35), 13-51.

Barbon, N. (1934). Discourse of trade, 1690. Reino Unido: Planograph Method.

Broadie, A. (Ed.) (2003). The Cambridge companion to the Scottish Enlightenment. Cambridge: Cambridge University Press.

Cantillon, R. (1950). Ensayo sobre la naturaleza del comercio en general. Buenos Aires: Fondo de Cultura Económica.

Cassirer, E. (1984). Filosofia de la Ilustración. México: Fondo de Cultura Económica. 
INVESTIGACIÓN SOBRE LA TRANSICIÓN ENTRE LAS DOCTRINAS MERCANTILISTAS Y EL NACIMIENTO DE LA

Colander, D., \& Landreth, H. (1994). History of economic thought. Boston: Houghton Mifflin Company.

Danford, J. W. (1990). David Hume and the Problem of Reason Recovering the Human Sciences. New Haven: Yale University Press.

Davanzati, B. (1997). A discourse upon coins. Londres: JD.

Griswold, C. L. (1999). Adam Smith and the virtues of enlightenment. Cambridge: Cambridge University Press.

Groenewegen, P. (2002). Eighteenth Century Economics. Londres: Routledge.

Haney, L. H. (1936). History of economic thought. Nueva York: The Macmillan Company.

Hume, D. (1752a). Of Money. McMaster University. Recuperado de http://socserv2. socsci.mcmaster.ca/ econ/ugcm/3113/ hume/money.txt

Hume, D. (1752b). On The Balance of Trade. McMaster University. Recuperado de http://socserv2.socsci.mcmaster.ca/ econ/ugcm/3113/hume/trade.txt

Hume, D. (1907). Essays: moral, political, and literary. Londres: Longmans, Green.

Hunt, E. K. (2015). History of economic thought: a critical perspective. Londres: M.E. Sharpe.

Levín, P. (1999). La Economía Política en el ocaso de su objeto. Enoikos, 15, 28-43.

Levin, P. (2003). Ensayo sobre la cataláctica. Buenos Aires: Facultad de Ciencias Económicas, Universidad de Buenos Aires.
Recuperado de http://www.economicas. uba.ar/wp-content/uploads/2015/11/Ensayo-sobre-la-Catal $\% \mathrm{C} 3 \% \mathrm{~A} 1 \mathrm{ctica} . \mathrm{pdf}$

Magnusson, L. (2015). The political economy of mercantilism. Nueva York: Routledge.

Marx, K. (1956). Historia crítica de la teoría de la plusvalía. Buenos Aires: Cartago.

Medema, S. G., \& Samuels, W. J. (2004). The history of economic thought: a reader. Londres: Routledge.

Mills, J. (2002). A critical history of economics. Basingstoke: Palgrave Macmillan.

Mun, T. (1664). La riqueza de Inglaterra por el comercio exterior. Discurso acerca del comercio de Inglaterra con las Indias Orientales. Recuperado de https://feubo. files.wordpress.com/2008/10/riqueza.doc

North, D. (1971). Discourses upon trade. Nueva York: Johnson Reprint Corporation.

Norton, D., (Ed.) (1995). The Cambridge Companion to Hume. Cambridge: Cambridge University Press.

Petty, W. (1769). A treatise of taxes [and] contributions. Londres: Brooke.

Rima, I. H. (2003). Development of economic analysis. Londres: Routledge.

Roll, E. (1969). Historia de las doctrinas económicas. México: Fondo de Cultura Económica.

Romero, J. L. (1987). Estudio de la mentalidad burguesa. Madrid: Alianza Editorial.

Roncaglia, A. (2006). The wealth of ideas: a history of economic thought. Cambridge: Cambridge University Press. 
Rubin, I. I. (1979). A history of economic thought. Londres: Ink links.

Schumpeter, J. A. (1954). History of economic analysis. Nueva York: Oxford University Press.

Screpanti, E., \& Zamagni, S. (2005). An outline of the history of economic thought. Oxford: Oxford University Press.

Steuart, J. (1767). An inquiry into the principles of political economy. Londres: A. Millar and T. Cadell. 